\title{
Juvenile Idiopathic Chondrolysis of the Hip
}

Startzman $\mathrm{AS}^{1^{*}}$, Hawkes $\mathrm{T}^{1}$ and Beterand $\mathrm{S}^{2}$

${ }^{1}$ Department of Orthopedics, Broward Health Medical Center, USA

${ }^{2}$ Department of Orthopedics, Georgia Regents University, USA

"Corresponding author: Startzman AS, Department of Orthopedics, Broward Health Medical Center, USA, Tel: 954-355-2091; Fax: 954-888-3448; E-mail: ashley.startzman@gmail.com

Rec date: Feb 24, 2016; Acc date: Jun 28, 2016; Pub date: Jun 30, 2016

Copyright: (c) 2016 Startzman AS, et al. This is an open-access article distributed under the terms of the Creative Commons Attribution License, which permits unrestricted use, distribution, and reproduction in any medium, provided the original author and source are credited.

\section{Abstract}

Introduction: Juvenile Idiopathic Chondrolysis the hip is a devastating disease. Early diagnosis is challenging. Clinicians must be aware of this rare but devastating diagnosis, as early diagnosis may improve prognosis.

Case presentation: An 11-year-old African American female presented to a pediatric orthopedic clinic with a 6month history of insidious onset left hip pain. Over 6-months her pain became more severe, and limited her ability to walk. The left hip was fixed in 40-degrees flexion, 20-degrees abduction, and 30-degrees of external rotation. New $\mathrm{x}$-ray findings consistent with idiopathic juvenile chondrolysis of the hip were present.

Discussion: First described in the early 1900s, chondrolysis of the hip was initially identified in patients following slipped capital femoral epiphysis. Later, patients with no clear etiology were diagnosed. Associations with trauma, burns, infection, and prolonged immobilization have also been described. The course is variable, with an acute phase lasting 6-16 months followed by a chronic stage of 3-5 years.

Conclusion: Idiopathic chondrolysis of the hip is a challenging diagnosis with devastating complications. Ultimately, most patients experience a spontaneous resolution of pain, develop early arthritis, and often arthrodesis.

Keywords: Chondrolysis; Hip chondrolysis; Pediatric hip; Idiopathic chondrolysis; Juvenile hip; Trauma hip; Slipped capital femoral epiphysis

\section{Introduction}

Juvenile Idiopathic Chondrolyisis of the hip is a devastating disease. Early identification of the disease process is sometimes challenging. Following diagnosis, there is ultimately a poor prognosis. Clinicians must be aware of this rare but devastating diagnosis, as early referral to a specialist may improve prognosis.

\section{Case Presentation}

An 11-year-old African American female presented to the pediatric orthopedic clinic 6 months after developing pain to her left hip. There was no trauma or inciting event, but a slow progression of pain that left her with diminished range of motion and mobility. She denied recent illness, fever, or travel. Her pain was exacerbated with movement, but still present at rest. There was no change in pain with time of day. No rash, or lymphadenopathy was present. There was no past medical or surgical history, and no family history of rheumatologic conditions or juvenile hip pain. She denied pain to other joints, and voiced no other complaints. Initially she was treated by her pediatrician, who ordered an $\mathrm{x}$-ray that revealed no abnormal pathology. Disease progression eventually left her with pain so severe that walking was challenging and sometimes not possible.

On exam, the patient was well developed. Her spine, upper extremities and right lower extremity examinations were within normal limits. The left hip was fixed in 40 degrees flexion, 20 degrees abduction, and 30 degrees of external rotation. Pain was present with limited motion ( $>1$ degree) to the hip. No effusion was present. Her left knee, ankle, and foot were non-tender to palpation or with range of motion. Her skin was intact with no evidence of erythema, ecchymosis, or warmth. She was neurovascularly intact. Her strength was decreased to the left lower extremity secondary to poor effort and left hip pain. She ambulated with an antalgic gait, and was able to apply only limited pressure to her left hip. She used the assistance of one crutch to ambulate.

New x-rays (Figures 1 and 2) revealed findings consistent with idiopathic juvenile chondrolyis of the hip including decreased joint space, sclerosis, and femoral and acetabular subchondral changes. An MRI of her hip demonstrated chondrolysis.

The patient had a trail of non-steroidal anti-inflammatory drugs (NSAIDs) and physical therapy. One month later, she described persistent and unrelenting pain. A steroid injection under fluoroscopic guidance was performed (Figure 3). While under general anesthesia for fluoroscopic guided intra-articular steroid injection, hip motion was improved. The left hip was able to be extended, adducted, and internally rotated to neutral, but not past this point. $80 \mathrm{mg}$ depomedrol and $3 \mathrm{cc}$ of $1 \%$ lidocaine were injected successfully to her left hip.

She was then instructed to be weight bearing as tolerated and referred back to physical therapy. At follow up one month after injection, she reported significant decrease in pain. However, on examination her hip remained to be fixed in an extended, abducted, and externally rotated position. She had resumed ambulating, but still required the assistance of one crutch. 


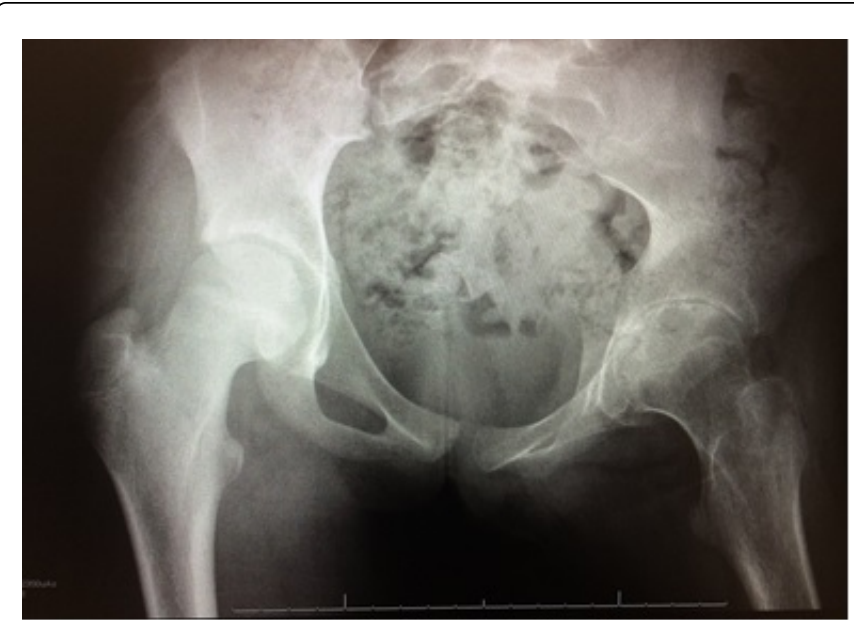

Figure 1: AP Pelvis X-ray 6 months after initial presentation to pediatrician demonstrating left hip condrolysis with joint space narrowing and osteopenia of the left femur and acetabulum.

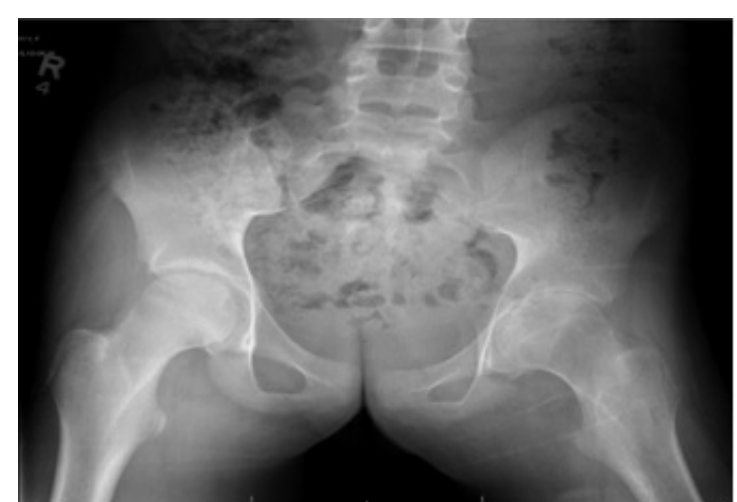

Figure 2: AP Pelvis X-ray 6 months after initial presentation to pediatrician demonstrating left hip condrolysis with joint space narrowing and osteopenia of the left femur and acetabulum.

Eight month post-injection follow up revealed great improvement in pain and the ability to ambulate without crutch assistance, but with a significant antalgic limp. The involved hip had no active or passive motion and still had a 40 degree flexion contracture, but was improved to neutral abduction/adduction, and was in 20 degrees of external rotation. 15 month follow up visit examination demonstrated a persistent 40 degree flexion contracture, atrophy to the thigh musculature, little pain with hip range of motion, and a non-antalgic gait.

\section{Discussion}

Idiopathic chondrolysis of the hip presents with an insidious, progressive onset of hip and groin pain. Pain is worse with range of motion, and eventually joint stiffness develops [1]. Patients will often ambulate with an antalgic gait [2].

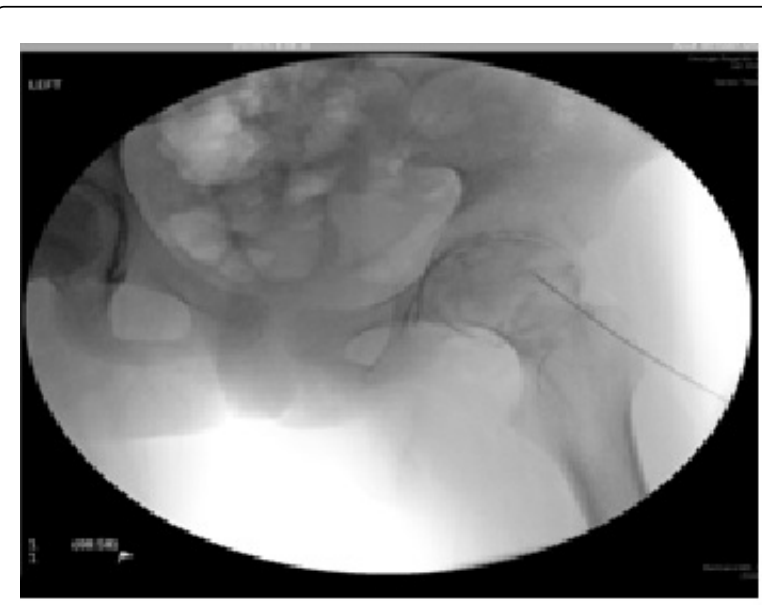

Figure 3: AP Left Hip Fluoroscopy during arthrogram procedure with injection of steroid.

Physical examination is the key to the diagnosis of idiopathic chondrolysis. Initially, patients will demonstrate the affected hip to be extended, adducted, and internally rotated [2]. This contrasts to other hip pathology including fracture, infection and joint effusion, which typically are associated with the hip in an abducted and externally rotated position. Later however, contractures will develop in an abducted, flexed, and externally rotated position [15]. Flexion contractures are more prevalent in cases of idiopathic chondrolysis as compared to patients with slipped capital femoral epiphysis [2].

Diagnosis can made with the assistance of radiographic studies. Early in the course of illness however, no changes on x-ray may be identified. Eventually, subchondral cysts, osteopenia, and osteophytes with sclerosis may be identified [2,3]. Chondrolysis is often limited to one joint. MRI is useful, and can demonstrate pathology sooner than plain radiographs including cartilage loss, muscle atrophy, and a small joint effusion with no enhancement of synovium.

Laboratory data will likely be within normal limits. Common tests may include joint aspiration, joint biopsy, white blood-cell count, sedimentation rate, rheumatoid factor, antinuclear antibody test, HLAB27 and CRP [2]. Intra-operative biopsies and exploration have revealed synovitis and cartilage loss [2].

\section{Etiology}

Chondrolysis of the hip was first described in the early 1900s. Initially, chondrolysis was identified in patients following slipped capital femoral epiphysis [1]. A presumed interruption in the synovial membrane of the hip joint was hypothesized to yield cartilage damage and death five to eight months following reduction of the slipped capital epiphysis [1]. Subsequently, literature reviews were performed in the 1970 s that documented an incidence of $1-28 \%$ of chondrolysis following slipped capital femoral epiphysis. Association with trauma and burns, infection, and prolonged immobilization have also been described. An autoimmune etiology has also been described. The initial autoimmune reaction to cartilage results in synovitis that further destroys cartilage. Patients with no clear etiology have also been 
diagnosed with chondrolysis [2,3]. Idiopathic chondrolysis typically occurs in black female adolescents. There is still no consensus regarding etiology [4-12].

\section{Treatment}

Treatment of chrondrolysis remains difficult, and few modalities provide adequate relief of pain for patients. Ambulation assistive devices such as crutches or a walker are often prescribed. Antiinflammatory medications and non-steroidal anti-inflammatory medications may provide some relief. Intra-articular injections and pain pumps may be of benefit. Prevention of contractures is key to treatment, with physical therapy often prescribed. Patient compliance with range of motion and therapy is challenging due to pain with activity [2].

Time from onset of pain to adequate improvement in pain is often 6-12 months. A small portion of patients may have spontaneous resolution of pain. Long-term pain however, is typically the case. [2] Patients with a disease process that yields joint anklyosis typically have resolution of pain [2]. Eventually, tenotomy, osteotomy, hip arthrodesis or early joint arthroplasty may be required [13-15].

\section{Conclusion}

Idiopathic chondrolysis of the hip is a challenging diagnosis with devastating complications. Early clinical suspicion must remain high among clinicians. Early diagnosis is important, as early treatment with physical therapy can ensure the patient maintains flexibility and avoids contractures. Ultimately, most patients experience a spontaneous resolution of pain with early arthritis and often arthrodesis.

\section{References}

1. Waldenstrom H (1996) On necrosis of the joint cartilage by epiphyseolysis capitis femoris. Clin Orthop Relat Res 322: 3-7.
2. Duncan JW, Nasca R J, Schrantz J (1979) Idiopathic Chondrolysis of the Hip. J Bone Joint Surg Am 61: 1024-1028.

3. Bleck E (1983) Idiopathic Condrolysis of the Hip. J Bone Joint Surg Am 65: 1266-1275

4. Pellicci PM, Wilson PD Jr (1979) Chondrolysis of the hips associated with severe burns A case Report. J Bone Joint Surg Am 61: 592-596.

5. Wenger DR, Mickelson MR, Ponseti IV (1975) Idiopathic chondrolysis of the hip. J Bone Joint Surg Am 57: 268-271.

6. Heppenstall RB, Marvel JP Jr, Chung SM, Brighton CT (1974) Chondrolysis of the hip. Clin Orthop Relat Res 103: 136-142.

7. Maurer RC, Larsen IJ (1970) Acute necrosis of cartilage in slipped capital femoral epiphysis. J Bone Joint Surg Am 52: 39-50.

8. del Couz Garci'a A, Fern'andez PL, Gonz'alez MP, Garcı'a AC, Gonz 'alez LR, et al. (1999) Idiopathic chondrolysis of the hip: long-term evolution. J Pediatr Orthop 19: 449-454.

9. Jacobs B (1972) Chondrolysis after epiphyseolysis. Inst Course Lect 21: 224-226.

10. Eisenstein A, Rothschild S(1976) Biochemical abnormalities in patients with slipped capital femoral epiphysis and chondrolysis. J Bone Joint Surg Am 58:459-467.

11. Jones BS (1971) Adolescent Chondrolysis of the Hip Joint. S Afr Med J 45: 196-202.

12. Moule NJ, Goulding JS (1974) Idiopathic chondrolyiss of the Hip. Clin Radiol 25: 247-251.

13. Provencher MT, Navaie M, Solomon DJ, Smith JC, Romeo AA, et al. (2011) Current Concepts Review Joint Chondrolysis. J Bone Joint Surg Am 93: 2033-2044.

14. Karol LA, Halliday SE, Gourineni P (2000) Gait and function after Intraarticular arthrodesis of the hip in adolescents. J Bone Joint Surg Am 82: $561-569$

15. Kay R (2006) Lovell and Winter's Pediatric Orthopaedics: Slipped Capital Femoral Epiphysis. (6thedition), Lippincott; Williams \& Wilkins. 